Methods A retrospective cohort study (database and clinical note review) to compare numbers of children within the population of 838573 in Lothian discharged on HETF from the single SE Scottish regional neonatal unit and the single SE Scottish regional paediatric unit, and their inter-relationships. All children and neonates discharged on HETF over the study period 1 July 2000-30 June 2011 were included. The fate of neonatal transfers requiring ETF to the paediatric centre was studied over the period of 1 July 2009-30 June 2011.

Results 485 Lothian children were discharged on HETF from the regional paediatric hospital (paediatric HETF) compared with 114 from the neonatal unit (neonatal HETF) over the same time period. Over the study period a rise in numbers requiring paediatric HETF was observed (average of 34 per year in 2000-2005 increasing to 55 per year in 2006-2011), however there was a decreasing number requiring neonatal HETF (average of 16 per year in 2000-2005 decreasing to 8 per year in 2006-2011). HETF was primarily used short term in the neonatal group for immaturity alone. During the 2 -year period of the study 7/2009-6/2011, 20 neonates were transferred to the regional paediatric unit on ETF; 11 (55\%) were subsequently discharged on HETF from the paediatric unit, but these neonatal transfers only accounted for $10 \%$ of the total of 103 paediatric HETF discharges.

Conclusion These novel data firstly demonstrate that the incidence of HETF usage on discharge from a UK paediatric regional centre continues to increase while that from the neonatal unit serving the same region is falling, and secondly that sick neonates transferred on ETF make up only a small number of incident paediatric HETF cases.

Competing interests None declared.

\section{PM0-072 A 1 YEAR TWO PHASE PROSPECTIVE PROJECT LOOKING AT NUTRITIONAL RISK IN REACTIVE VS ELECTIVE NASOGASTRIC ENTERAL FEEDING IN HEAD AND NECK CANCER PATIENTS UNDERGOING RADICAL (CHEMO)RADIOTHERAPY}

doi:10.1136/gutjnl-2012-302514b.72

${ }^{1} \mathrm{C}$ H Sheth, ${ }^{*}{ }^{1} \mathrm{~S}$ Sharp, ${ }^{2} \mathrm{C}$ Baughan, ${ }^{1} \mathrm{E}$ Walters. ${ }^{1}$ Department of Nutrition \& Dietetics, University Hospital Southampton NHS Foundation Trust, Southampton, UK; ${ }^{2}$ Department of Oncology, University Hospital Southampton NHS Foundation Trust, Southampton, UK

Introduction Pre 2009 head and neck cancer (HNC) patients requiring enteral tube feeding during radical (chemo)radiotherapy at our regional cancer centre were admitted reactively in a nutritional "crisis" and fed via a nasogastric(NG) tube. ${ }^{1}$ Audit resulted in a 1-year service improvement grant for a Specialist Dietitian to proactively support HNC patients "from pre-treatment until rehabilitation is complete". ${ }^{2}$ The aim of this 1-year (2009-2010) twophase prospective project was to reduce nutrition related crisis admissions, malnutrition and refeeding syndrome, thereby reducing length of hospital stay (LOS) from an average of 13 days ${ }^{1}$ while aiming to improve patient experience and outcome.
Methods Phase1 (9 months): All patients with HNC (squamous cell carcinoma) were included. Nutritional status (MUST score), \%weight loss and swallow ability was recorded for all patients before, during and after radiotherapy treatment. Patients were admitted reactively with inadequate nutritional intake and/or $5 \%$ weight loss. Interim review of phase 1 highlighted that oral cavity, oropharyngeal, nasopharyngeal, hypopharyngeal carcinomas and unknown primary tumours were at nutritional risk from weeks 2 and 3 of radiotherapy, leading to reactive admissions for NG feeding. Phase 2 ( 3 months) involved patients with these tumours admitted electively for NG feeding in week 3 radiotherapy. All admitted patients were followed up for 6 months.

Results Refeeding risk, number of days until nutritionally fit and LOS were all significantly reduced in phase 2 compared to phase 1 .

Conclusion This data demonstrates that when appropriately funded, a specialist dietetic service working as part of a multidisciplinary team in HNC, by electively admitting high nutritional risk patients for NG feeding, can significantly reduce clinical risk and costs. As a result of clinical benefits and cost savings our Trust made the service improvement funding substantive.

Competing interests None declared.

\section{REFERENCES}

1. Sheth CH, Sharp S, Walters E. A two year audit of enteral feeding in head and neck cancer patients receiving radiotherapy or chemoradiotherapy treatment at a UK Cancer Centre. 2011. Article submitted for publication.

2. National Institute for Clinical Excellence. Improving Outcomes for Head \& Neck Cancer. London: NICE, 2004.

\section{PM0-073 POST-OPERATIVE ENTERAL NUTRITION AND RECIRCULATION OF JEJUNAL EFFLUENT IN THE MANAGEMENT OF A PARADUODENAL HERNIA: A CASE REPORT}

doi:10.1136/gutjnl-2012-302514b.73

${ }^{1} \mathrm{D}$ Mcgrogan, ${ }^{* 1} \mathrm{~S}$ McCain, ${ }^{1} \mathrm{~A}$ Harris, ${ }^{2} \mathrm{~K}$ McCallion. ${ }^{1}$ General Surgery, Southern Health and Social Care Trust, Craigavon, UK; ${ }^{2}$ General Surgery, Southeastern Health and Social Care Trust, Belfast, UK

Introduction Short gut secondary to surgical resection often requires post-operative total parenteral nutrition. We present a case of ischaemic gut secondary to a paraduodenal hernia. Post-operative nutritional requirements were met using enteral feeding via a feeding jejunostomy and recycling end jejunostomy effluent.

Methods Case: A female presented with abdominal pain and metabolic acidosis. A double contrast abdominal computer topography scan revealed necrotic small bowel secondary to an internal hernia into the right paraduodenal fossa. She was found to have $180 \mathrm{~cm}$ of infarcted jejunum and ileum. After resection of this segment an end jejunostomy was formed along with a closed mucus fistula from proximal ileum. $50 \mathrm{~cm}$ proximal to this a feeding jejunostomy/ ileostomy was constructed. Early jejunal feeding with elemental feed was commenced on day 1 . Loperamide and codeine were added to slow intestinal transit and promote absorption. On day 5, bile excreted via her jejunostomy was recycled via her feeding

Abstract PM0-072 Table 1

\begin{tabular}{|c|c|c|c|c|c|}
\hline & $\begin{array}{l}\text { Total number } \\
\text { of patients }\end{array}$ & $\begin{array}{l}\text { Total patients } \\
\text { admitted }\end{array}$ & $\begin{array}{l}\% \text { Patients at risk of } \\
\text { refeeding syndrome on admission }\end{array}$ & $\begin{array}{l}\text { Average number of days from admission } \\
\text { until full nutritional requirements met }\end{array}$ & Average LOS days \\
\hline Audit $^{1}$ (2006-2008) & 223 & 32 & 94 & $11(S D \pm 4.9)$ & $13(S D \pm 5.1)$ \\
\hline Phase 1 & 83 & 32 & 75 & $8.2(S D \pm 5.4)$ & $9.3(\mathrm{SD} \pm 6.1)$ \\
\hline Phase 2 & 23 & 9 & 33 & $3.2(S D \pm 2.7)$ & $4.3(S D \pm 5.2)$ \\
\hline p Value (phase 1 \& 2) & & & $0.04^{*}$ & $0.01 * *$ & $0.0065^{* *}$ \\
\hline
\end{tabular}

*Fisher's exact test, two-tailed.

**T-test, unpaired, two-tailed. 
jejunostomy. Her enteral feed was changed from elemental to modular on day 8 and sandostatin commenced. Her nutritional markers improved on this regime and despite having only $50 \mathrm{~cm}$ of jejunum proximal to her stoma, outputs were low and her electrolytes remained normal. She put on weight post-operatively and proceded to closure of her stomas at 6 months.

Results N/A.

Conclusion Discussion: Internal hernias are rare and commonly present with small bowel obstruction. Paraduodenal hernias are the most common form and can be either left or right sided. Normal small intestinal length varies between about $275 \mathrm{~cm}$ to $850 \mathrm{~cm}$ with nutritional or fluid supplementation required for patients with $<200 \mathrm{~cm}$. This lady had a post-operative bowel length of $225 \mathrm{~cm}$. The majority of gastrointestinal secretions are reabsorbed in the upper jejunum and patients with jejunostomies often have large volume stomal output. We used loperamide and codeine to slow intestinal transit, aid absorption and prevent fluid and electrolyte imbalance. Limited reports of bile-jejunostomy recycling were found in the literature and to our knowledge no cases have been reported of patients with short bowel being managed using recirculation of jejunal effluent and enteral nutrition in isolation. In this case, jejunostomy fluid was collected and immediately recycled via a feeding ileostomy eliminating the need for parenteral nutrition. Success was shown by improvement in nutritional status and weight gain.

Conclusion This case exemplifies the effective use of jejunostomy output recycling to reduce water and electrolyte loss and aid fat absorption in a patient with short gut thus preventing the use of parenteral nutrition.

Competing interests None declared.

\section{PM0-074 A 10 YEAR RETROSPECTIVE REVIEW OF NASOGASTRIC TUBE USE IN A DISTRICT GENERAL HOSPITAL}

doi:10.1136/gutjnl-2012-302514b.74

E Tash, ${ }^{*}$ A Cartwright, L Steel, J Subhani. Department of Gastroenterology, Basildon and Thurrock University Hospitals NHS Foundation Trust, London, UK

Introduction A Nasogastric tube (NGT) is a commonly used device for administration of enteral feeding. It may be used as a bridge to a more definitive procedure (eg, Percutaneous Endoscopic Gastrostomy; PEG) or until patient recovery. The NPSA estimates that "thousands of NGT are placed each day" throughout the UK. ${ }^{1}$ However, following a literature review we were unable to find any outcome data for this procedure.

Methods We performed a retrospective review of our nutrition database at Basildon and Thurrock University Hospitals NHS Foundation Trust, that serves a population of 375000 , for all patients who had an NGT inserted between years 2001 and 2011 and analysed the insertion reason and outcome.

Results Over a 10-year period, 2526 patients underwent 2715 episodes of NGT insertions, with a male to female ratio of $49.5 \%$ and $50.5 \%$, respectively. The total number of feeding days was
50584 , with a median and mean length of insertion of 10 and 18.7 days, respectively. 189 patients required a repeat NG feeding episode, either within the same or subsequent hospital admission. The referral reasons and outcome data are shown in the Abstract PMO-074 table 1 below.

Conclusion Data analysis revealed that two thirds of all NGT insertions were for patients with dysphagia secondary to a neurological condition, mechanical obstruction or artificial ventilation. Despite these patients being unwell and high risk for complications $46.9 \%$ were able to restart oral feeding. However, almost an equal number died while receiving NGT enteral feeding. $2.7 \%$ of our cohort were discharged home with some form of long term enteral feeding; either via a PEG or longterm NGT.

Competing interests None declared.

\section{REFERENCE}

1. Patient safety alert NPSA/2011/PSA002: reducing the harm caused by misplaced nasogastric feeding tubes in adults, children and infants. 2011. Supporting Information.

\section{PMO-075 30-DAY MORTALITY RISK FACTORS FOR PERCUTANEOUS ENDOSCOPIC AND RADIOLOGICALLY-INSERTED GASTROSTOMY}

doi:10.1136/gutjnl-2012-302514b.75

F M Laskaratos, ${ }^{*}$ M Walker, V Wojciechowska, A Jenkins. Department of Gastroenterology, Princess Royal University Hospital, London, UK

Introduction Gastrostomy insertion is of benefit in selected patients, ${ }^{1}$ but 30 -day mortality is as high as $54 \%$ in some patient groups. ${ }^{2}$ Risk factors associated with early mortality include advanced dementia, age $>75$ years, serum albumin $<30 \mathrm{~g} / \mathrm{l}$, C-reactive protein $>10 \mathrm{mg} / \mathrm{l}$, cardiovascular and respiratory comorbidities. ${ }^{34}$ The Sheffield scoring system has been developed to identify patients at risk of early death. ${ }^{5}$ The current study examines risk factors associated with 30 day mortality in a cohort of patients who underwent percutaneous endoscopic gastrostomy (PEG) or radiologically-inserted gastrostomy (RIG) in a district general hospital over a 12-month period.

Methods A retrospective review was made of case notes of all patients who underwent a PEG $(n=26)$ or RIG $(n=16)$ insertion in the period July 2010-July 2011. PEG/RIG re-insertions were excluded. Demographic, clinical and biochemical data were analysed. Results The main indications for a PEG/RIG insertion were cerebrovascular accident $(n=14)$, chronic neuromuscular disease $(n=10)$, oropharyngeal malignancy $(n=8)$, intracerebral pathology other than stroke $(n=6)$, cognitive impairment $(n=2)$, ICU neuropathy $(n=1)$ and somatisation disorder $(\mathrm{n}=1)$. The overall 30-day mortality was $7 / 42(16.7 \%)$. The main risk factors associated with 30-day mortality were age $>75$ years $(p<0.05)$ and cardiovascular co-morbidities $(\mathrm{p}<0.01)$. The serum albumin, C-reactive protein and respiratory comorbidities were not associated with early mortality. There was no correlation between 30-day mortality and Sheffield score.

\section{Abstract PM0-074 table 1}

\begin{tabular}{|c|c|c|c|c|c|}
\hline Reason for referral & Total episodes & Percentage of total (\%) & Outcome of NG feeding & Total episodes & Percentage of total $1 \%$ \\
\hline Dysphagia & 1689 & 62.2 & Restart oral feeding & 1272 & 46.9 \\
\hline Malnutrition & 635 & 23.4 & Died & 1208 & 44.5 \\
\hline Post operative GI tract & 266 & 9.8 & Transferred out of area & 157 & 5.8 \\
\hline Miscellaneous* & 69 & 2.5 & Discharged home on enteral feeding & 74 & 2.7 \\
\hline Post operative non Gl tract & 42 & 1.5 & Self discharged & 2 & 0.1 \\
\hline Increased nutritional & 25 & 0.9 & Missing data & 2 & 0.1 \\
\hline
\end{tabular}

requirements

${ }^{*}$ Miscellaneous includes missing data (19 and 0.7); intestinal obstruction (16 and 0.6); intestinal failure (8 and 0.3); malabsorption (4 and 0.2) and NG feed during the course of an episode of acute pancreatitis (22 and 0.8$)$ 